\title{
LX. The theoretical evaluation of the ratio of the specific heats of a gas
}

\author{
J.H. Jeans B.A.
}

To cite this article:J.H. Jeans B.A. (1901) LX. The theoretical evaluation of the ratio of the specific heats of a gas , Philosophical Magazine Series 6, 2:12, 638-651, DOI: 10.1080/14786440109462728

To link to this article: http://dx.doi.org/10.1080/14786440109462728

册 Published online: 08 Jun 2010.

Submit your article to this journal $[\pi$

Џ Article views: 3

Q View related articles $\square$ 
It appears that the metal by its own attraction for the charge on the ion tends to neutralize the effect of the velocity of impact; so that, with the current in the steady state, the metal exerting the greater attraction for the charge it receives possesses the smaller drop of potential. The series of metals arranged according to the magnitude of the cathode drop would be thus the reverse of that for the anode drop.

We should expect the relatively large variation in both cathode and anode drops when the electrodes become tarnished to be due to the same influences; but here the results are exactly the reverse of what we should predict from the contact-potential series. According to Lord Kelvin *, "dry oxide of copper is resinous to copper in contact with it, and dry oxide of zinc is resinous to zinc in contact with it, just as copper is resinous to zinc in contact with it." While we would predict then, that, by tarnishing, the drop at the anode should decrease, I have found by experiment (l.c.) that there is invariably an increase in the drop at the anode, Warburg (l.c.) having previously found that the drop at the cathode decreases.

Physical Laboratory,

University of Nebraska, Lincoln.

LX. The Theortical Evaluation of the Ratio of the Specific Heats of a Gas. By J. H. JeAns, B.A., Fellow of Trinity College, and Isaac Newton Student in the University of Cambridge $\dagger$.

\section{Introduction.}

$\S 1 . T$ TE Maxwell-Boltzmann theorem on the Partition of Energy leads, as is well known, to a value of $\gamma$ which is not in accordance with observed values. In a recently published paper $\ddagger I$ have suggested that an escape from the conclusions of this theorem might be found by taking account of the interaction between matter and xther; and it. was shown that even a small interaction might entirely modify the conclusions of the theorem. In the present paper an attempt is made to work out in greater detail the nature of these modifications, and, in particular, to examine whether it is possible, in this way, to obtain a theoretical evaluation of $\gamma$ which shall be in agreement with observed values.

- Lord Kelvin, Phil. Mag. July 1898, p. 82.

† Communicated by Prof. J. J. Thomson, F.R.S.

\pm "The Distribution of Molecular Energy," Phil. Trans. cxcvi. p. 397 . 
Experimentally the value of $\gamma$ is best found indirectly by observation of the velocity of sound; and this introduces a further complication into the question. This complication arises as follows.

In arriving at Laplace's value for the velocity of sound in a gas $*$, it is assumed that the ratio of the two specific heats of a gas has a definite value, $\gamma$, which depends solely upon the nature of the gas. This, as can easily be verified, amounts to assuming that the mean internal energy of a molecule bears, at every instant, a constant ratio $\frac{2}{3(\gamma-1)}-1$ to the mean
energy of translation.

Now, after the lapse of infinite time, a gas tends to assume a steady state in which the two mean energies may, perhaps, be legitimately supposed to bear to one another this constant ratio; but the case is different with a gas in which the value of one of the quantities in question is continually caused to vary owing to the passage of a wave of sound. For the mechanism by which the balance of energy is adjusted cannot be supposed to work with infinite rapidity, so that the ratio in question will never have the actual value which must be assigned to it in order to arrive at the Laplacean velocity of sound. The question as to whether or not this want of steadiness in the ratio of the two energies is sufficient to influence appreciably the transmission of sound, is therefore seen to be one requiring investigation.

\section{General Theory.}

\$2. Let us, for the sake of simplicity, consider a gas of which the molecules possess only one kind of freedom in addition to the freedom to move in space. Let the mean energy of translation in space be denoted by $\mathbf{E}$, and the mean total energy by $\mathbf{E}+\mathbf{F}$, the energy $\mathbf{F}$ arising from the remaining kind of freedom, which may be either a rotation or an internal vibration.

The quantities $\mathrm{E}$ and $\mathrm{F}$ will be capable of variation owing to the transfer of energy which is effected by collisions between the molecules of the gas. In order to obtain sufficiently general results we find it necessary to imagine a second path for the transfer of energy, namely æther-vibrations. Regarding the molecule as an electromagnetic system, the vibrations or rotation of the system will send out electromagnetic waves into the æther, and collision of the two molecules will also send out a system of irregular electromagnetic waves or

* Lord Rayleigh, ‘'Theory of Sound,' $\$ 246$. 
"pulses." The energy of these waves will be either partially or entirely absorbed by the other molecules of the gas, either by forcing vibrations in them, or by affecting their velocities of translation or rotation; and in this way we have a second path for the transfer of energy. If this absorption is complete, the total mean energy $\mathbf{E}+\mathbf{F}$ will romain constant throughout, and a steady state will be possible. If the absorption is only partial, a steady state will only be possible if we suppose energy introduced into the gas by some external agency, of amount sufficient to compensate that which is lost by the radiation of waves.

If the law of distribution of $\mathrm{E}$ and $\mathrm{F}$ among the molecules of the gas is known, it will be possible to calculate the two transfers caused respectively by collisions and electromagnetic waves. The rate at which $F$ increases owing to collisions will be some function of $\mathrm{E}$ and $\mathrm{F}$, which will for the present be denoted by $\phi(\mathbf{E}, \mathrm{F})$; the rate at which $\mathrm{F}$ increases owing to radiation and absorption may similarly be taken to be $\psi(E, F)$. We therefore have, for the total change in $F$,

$$
\frac{d \mathrm{~F}}{d t}=\phi(\mathrm{E}, \mathrm{F})+\psi(\mathrm{E}, \mathrm{F}) . . . .
$$

There must be at least one steady state; let the corresponding root of

be

$$
\begin{aligned}
\phi(\mathrm{E}, \mathrm{F}) & +\psi(\mathrm{E}, \mathrm{F})=0 \\
\mathrm{~F} & =f(\mathrm{E}) .
\end{aligned}
$$

When the reaction with the rther is of zero amount, equation (3) will reduce to the expression of the MaxwellBoltzmann law; but a very small reaction may, as was pointed out in the paper previously referred to, suffice to alter entirely the form of this equation. The value of $\gamma$ is now given by

$$
\frac{d \mathrm{E}}{d(\mathrm{E}+\mathrm{F})}=\mathbf{3}_{2}(\gamma-1)
$$

or

$$
\gamma=1+\frac{2}{3\left(1+f^{\prime}(\mathbf{E})\right)}, \ldots . . .
$$

where $f^{\prime}(\mathrm{E})$ stands for $d f(\mathrm{E}) / d \mathrm{E}$.

This value of $\gamma$ is not restricted to being one of the series given by the formula $1+2 / n$, and is, moreover, capable of variation with the temperature.

$\S 3$. The right-hand member of equation (1) contains as a 
factor either $\mathrm{F} \rightarrow f(\mathbf{E})$, or else some power of this fuctor, $\|^{\circ}$ else some other function of this factor which ranishes when the factor is put equal to zero. In order that a simply periodic variation in $\mathbf{F}$ and $\mathbf{E}$ may be possible ( $i$. $e$. in order that sound may bo propagated in the gas) the first of these alternatives must be the true one. In this case, assuming the values of $E$ and $F$ to vary only slightly from their equilibrium values $\mathrm{E}_{0}, \mathrm{~F}_{0}$, equation (1) may be written in the form

$$
\frac{d \mathrm{~F}}{d t}=-\chi\left(\mathrm{E}_{0}\right)[\mathrm{F}-f(\mathrm{E})], \quad \cdot \quad \cdot \cdot
$$

where $\chi\left(\mathrm{E}_{0}\right)$ is some function of $\mathrm{E}_{0}$ which is positive if the gas tends to return to the steady state in which $\mathrm{E}=\mathrm{E}_{0}, \mathrm{~F}=\mathrm{F}_{0}$.

Assuming as a general solution

$$
\begin{aligned}
& \mathrm{E}=\mathrm{E}_{0}+\mathrm{E}_{1} e^{i p t}, \\
& \mathrm{~F}=\mathrm{F}_{0}+\mathrm{F}_{1} e^{i p t},
\end{aligned}
$$

we find from (5) the equation

$$
i p \mathrm{~F}_{1}=-\chi\left(\mathrm{E}_{0}\right)\left[\mathrm{F}_{1}-f^{4}\left(\mathrm{E}_{0}\right) \mathrm{E}_{1}\right] \text {; }
$$

or, what is the same thing,

where

$$
\mathrm{F}_{1}=\beta \mathrm{E}_{1}, \quad \cdot \quad \cdot \quad \cdot \quad \cdot \quad \cdot
$$

$$
\beta=f^{\prime}\left(\mathrm{E}_{0}\right) /\left(1+\frac{i p}{\chi\left(\mathrm{E}_{0}\right)}\right) \ldots . . .
$$

$\$ 4$. Let $\approx, \rho$ be the pressure and density of the element of gas under consideration, and let us assume as a solution for $\varpi, \rho$ the values

$$
\begin{aligned}
& \varpi=\varpi_{0}+\varpi_{1} e^{i p t}, \quad . \quad . \quad . \quad . \quad . \quad . \\
& \rho=\rho_{0}+\rho_{1} e^{i p t} . \quad . \quad . \quad . \quad . \quad .
\end{aligned}
$$

The quantities $\approx, \rho$, and $E$ are comnected by the relation

$$
\varpi=\lambda E \rho, \cdot \cdot \cdot \cdot \cdot \cdot \cdot \cdot
$$

where $\lambda$ is a constant. Substituting the assumed values of $\varpi, \rho$, and $\mathrm{E}$, we find at once the relation

$$
\frac{\varpi_{1}}{\varpi_{0}}=\frac{\mathrm{F}_{1}}{\mathrm{E}_{0}}+\frac{\rho_{1}}{\rho_{0}} . . . . . .
$$

We further suppose that no heat enters or leaves the element in question, so that the total energy of the element 
remains constant. This gives the equation*

$$
\frac{3}{2}(\delta \mathrm{E}+\delta \mathrm{F})+\rho \mathrm{E} \delta\left(\frac{1}{\rho}\right)=0,
$$

in which $\delta$ denotes excess above the mean value. Substituting for $\mathrm{E}, \mathrm{F}$, and $\rho$, this gives

$$
\frac{3}{2}\left(\mathrm{E}_{1}+\mathrm{F}_{1}\right)-\frac{\mathrm{E}_{0} \rho_{1}}{\rho_{0}}=0:
$$

or, by equation (6),

$$
\frac{3}{2}(1+\beta) \frac{\mathrm{E}_{1}}{\mathrm{E}_{0}}-\frac{\rho_{1}}{\rho_{0}}=0 .
$$

Combining this with equation (11), we find

where

$$
\frac{\varpi_{1}}{\varpi_{0}}=\Gamma \frac{\rho_{1}}{\rho_{0}}, \quad . \quad . \quad . \quad . \quad .
$$

$$
\begin{aligned}
\Gamma & =1+\frac{2}{3(1+\beta)} \cdot \ldots . . . \\
& =1+\frac{2}{3\left\{1+f^{\prime}(\mathrm{E}) /\left(1+\frac{i p}{\chi(\mathrm{E})}\right)\right\}} .
\end{aligned}
$$

If we had supposed the gas to obey the ordinarily-assumed equation for adiabatic motion, viz.

we should have obtained

$$
\approx \propto \rho \gamma,
$$

$$
\frac{\sigma_{1}}{\sigma_{0}}=\gamma \frac{\rho_{1}}{\rho_{0}}
$$

$\S 5$. Comparing this with equation (12), we see that the quantity $\Gamma$ given by equation (13) is a generalized form of the usual quantity $\gamma$. When the variation in $\mathrm{E}$ is infinitely slow $(i$. $e$. when $p=0$ ), the ratio between $\mathbf{F}$ and $\mathbf{E}$ is always the equilibrium ratio, and $\Gamma$ becomes identical with $\gamma$. So that we find, in this case, that equations (14) and (5) become identical. When, on the other hand, the variation in $\mathrm{E}$ is of infinite rapidity, the value of $\mathrm{F}$ is unaffected by variations in $\mathrm{E}$, and maintains a constant value corresponding to the mean value of $\mathrm{E}$. For this reason, the gas will behave like a gas of which the molecules possess only the three translational degrees of freedom, $i$. e. like a gas for which $\gamma=1 \frac{2}{3}$.

* Boltzmann, Vorlesungen über Gastheorie, i. p. 56. 
In accordance with this we find, on putting $p=\infty$ in (14), that we obtain $\Gamma=1 \frac{2}{3}$.

\section{The Propagation of Sound.}

$\S 6$. Independently of any assumptions about the internal energy of the molecules, the equation of propagation of sound is *

$$
\frac{d^{2} \phi}{d t^{2}}=\frac{\delta \varpi}{\partial \rho} \frac{d^{2} \phi}{d x^{2}}
$$

where $\phi$ is the velocity-potential, and $\delta$, as before, denotes excess above mean value.

Assuming $\phi=\phi_{1} e^{i p t}$, we find

$$
\left(p^{2}+\frac{\varpi_{1}}{\rho_{1}} \frac{d^{2}}{d x^{2}}\right) \phi_{1}=0,
$$

or, by equation (12),

$$
\left(p^{2}+\frac{\Gamma \varpi_{0}}{\rho_{0}} \frac{d^{2}}{d x^{2}}\right) \phi_{1}=0 . . . \quad . \quad .
$$

The solution for a wave travelling along the positive axis of $x$ is

$$
\phi_{1}=\mathrm{A} e^{i p\left(t-\frac{x}{\mathrm{v}}\right)}, \text {. . . . . . }
$$

where $\frac{1}{\nabla^{2}}=\frac{\rho_{0}}{\Gamma \varpi_{0}}$.

This gives

$$
\nabla=a \sqrt{\Gamma},
$$

where $a$ is the Newtonian velocity of sound.

The difference between this solution and that usually found lies in the occurrence of terms in $i p / x(\mathbb{E})$. From equation (14) we obtain

where

$$
\Gamma^{-\frac{1}{2}}=\gamma^{-\frac{1}{2}}(\alpha-i \beta), \quad . \quad . \quad . \quad . \quad .
$$

$$
\begin{aligned}
& \alpha=1-\left\{\frac{p}{\chi(\mathrm{E})}\right\}^{2}\left\{\frac{f^{\prime}(\mathrm{E})}{3 \gamma\left(1+f^{\prime}(\mathrm{E})\right)^{3}}+\frac{\left(f^{\prime}(\mathrm{E})\right)^{2}}{6 \gamma^{2}\left(\overline{1}+f^{\prime}(\mathrm{E})\right)^{4}}\right\}, \\
& \beta=\frac{p}{\chi(\mathrm{E})} \frac{f^{\prime}(\mathrm{E})}{3 \gamma\left(1+f^{\prime}(\mathrm{E})\right)^{2}},
\end{aligned}
$$

as far as squares of $p / \chi(\mathrm{E})$.

The functions $\chi(\mathrm{E}), f^{\prime}(\mathrm{E})$ will be positive $(c f . \S 8)$, so that as far as terms in $p^{2}, \beta$ will always be positive, an $d$ a less than unity.

* Rayleigh, 'Theory of Sound,' $\$ 244$. 
Equation (16) now becomes

$$
\phi=\mathrm{A} e^{i p\left(t-\frac{x a}{a_{\sqrt{V}}}\right)} e^{-\frac{x \mu \beta}{a_{\sqrt{ } V} V}} . . . . .
$$

It appears from this that the value of $\gamma$ deduced from experiments on the velocity of sound will not be the true $\gamma$, but will be

$$
\gamma / \alpha^{2} \text {. }
$$

This, however, only differs from $\gamma$ by terms depending on the square of $p / \chi(\mathrm{E})$. The effect of the first order terms is the introduction of a real exponential term into the expression for $\phi$, corresponding physically to a damping of the sound. The amount of the damping, as also of that due to viscosity, is proportional to $p^{2}$; so that the effect of the "lag" in the value of $\mathrm{F}$ may be regarded as an apparent increase in the coefficient of viscosity.

Lord Rayleigh * shows that corresponding to a small coefficient of viscosity $\mu$, the coefficient of decay (measured per unit length along the path of the wave) is

$$
\frac{2 \mu p^{2}}{3 p_{0} \mathrm{~V}^{3}}
$$

while the coefficient of decay due to the "lag" in F has been shown to be

$$
\frac{p^{2} f^{\prime}(\mathrm{E})}{3 \gamma \mathrm{V} \chi(\mathrm{E})\left(\left(1+f^{\prime}(\mathrm{E})\right)^{2}\right.}
$$

Thus the apparent value of $\mu$ will be

$$
\mu\left\{1+\frac{\varpi_{i} f^{\prime}(\mathrm{E})}{2 \mu \chi(\mathrm{E})\left(1+f^{\prime}(\mathrm{E})\right)^{2}}\right\} . . . .
$$

Since $a$ only differs from unity by terms depending on the square of $p / \chi(\mathrm{E})$, we see that at first the apparent value of $\gamma$ remains unaltered being equal to the true value. As soon as squares of $p / \chi(\mathrm{E})$ become appreciable, the apparent value of $\gamma$ begins to increase, and ultimately, when $p / \chi(\mathrm{E})$ becomes infinite, the apparent value of $\gamma$ will be $1 \frac{2}{3}$.

\section{The Lag in Rotatory Energy in Air.}

$\S 7$. As an illustration of the foregoing theory, let us examine the effect of the lag in the rotation of molecules of air, the molecule being regarded for the purpose as a rigid body of which the shape is that of a solid of revolution. The quantity $\mathrm{F}$ is now the energy of the rotation of the molecule,

$$
\text { * 'Theory of Sound,' } § 346 \text {, equation (7). }
$$


the rotation about the axis of symmetry being excluded. We have now

$$
\begin{aligned}
\gamma & =12, \\
f(\mathrm{E}) & =2 \mathrm{E},
\end{aligned}
$$

if we assume the rotation to be uninfluenced by reaction with the surrounding æther, and

$$
f^{\prime}(\mathrm{E})=\frac{2}{3},
$$

while equation (5) becomes

$$
\frac{d \mathrm{~F}}{d t}=-\chi\left(\mathrm{E}_{0}\right)(\mathrm{F}-2 \mathrm{E} \mathrm{E}) .
$$

There is considerable difficulty in forming a reliable estimate of the value of $\chi\left(\mathrm{E}_{0}\right)$. The only case in which $I$ have succeeded in calculating such a value is the case in which the molecules are supposed to consist of geometrically perfect spheres (of radius $\mathbf{R}$ and mass $m$ ), in which the centre of gravity is at a small distance $r$ from the geometrical centre. In this case the value is

$$
\chi(\mathrm{E})=\frac{16 r^{2} \mathrm{R}^{2} \rho}{3 m k^{2}} \sqrt{\frac{2 \pi}{3 m} \mathrm{E}}
$$

where $k$ is the radius of gyration*.

The factor which depends on the geometrical structure of the molecule is $r^{2} R^{2} / k^{2}$. For a molecule in which the divergence from spherical symmetry is not small, we may probably, without error as regards order of magnitude, replace $r^{2} / k^{2}$ by unity. This gives, as an approximate value,

$$
\chi(\mathrm{E})=8 \mathrm{R}^{2} n c
$$

where $n$ is the number of molecules per cu. centim., and $c^{2}$ is the mean-square of their velocity. Taking $R=10^{-8} \mathrm{~cm}$., $n=10^{20}, c=4 \times 10^{4}$, we obtain

$$
\chi(\mathrm{E})=3 \times 10^{9} .
$$

In the same units as before, we may take $\mu=\cdot 00019$, $\varpi_{0}=\rho_{0} \mathrm{~V}^{2} / \gamma=8 \cdot 6 \times 10^{5}$, and the value of expression (19) becomes

$$
1 \cdot 2 \mu \text {. }
$$

The numerical estimate of $\chi(E)$ is so vague that very little importance can be attached to the actual result obtained.

* The calculation is too lengthy to be reproduced here. I have indicated the method elsewhere (Phil. Trans. cxcri. p. 399).

Phil. Mag. S. 6. Vol. 2. No. 12. Dec. 1901. $2 \mathrm{U}$ 
The result seems, however, to suggest that part of the damping of sound in air may be assignable to causes other than pure viscosity.

The value of $\alpha$ is roughly

$$
\alpha=1-\frac{1}{20}\left\{\frac{p}{\chi(\mathbf{H})}\right\}^{2},
$$

so that $\chi(\mathrm{E})$ might vary largely from our numerical estimate of equation (20) before any appreciable difference could be expected between the true and apparent values of $\gamma$.

\section{Subsidiary Degrees of Freedom.}

\$8. We have supposed, in dealing with rotatory energy in the last section, that the energy of rotation is, in the steady state, equal to the value which Boltzmann's theorem would assign to it. In other words, we have assumed that in equation (1) the term $\phi(E, F)$ is so large as to outweigh the term $\psi(E, F)$.

In addition to degrees of freedom of this kind, there may be other degrees of freedom, for which $\phi(\mathrm{E}, \mathrm{F})$ will be comparable with $\psi(\mathrm{E}, \mathrm{F})$, the transfer of energy being determined as much by the æether-reactions as by collisions. It is convenient to refer to these two kinds of degrees of freedom as "principal" and "subsidiary" respectively.

Let us suppose tbat a molecule possesses, in addition to its freedom to move in space, $n$ "principal " degrees of freedom and $m$ " subsidiary" degrees of freedom, the energy of each of the latter being supposed to have the same mean value $F$ [e.g., for a single internal vibration, $m=2$; for a single spectral line which can be separated into $s$ lines, we probably have $m=2 s$.

If we ignore the "lag" in the energy of the principal degrees of freedom, we may write (cf. equation 14)

$$
\Gamma=1+\frac{2}{3+n+3 m f^{\prime}(\mathrm{E}) /\left(1+\frac{i p}{\chi(\mathrm{E})}\right)}, \quad .
$$

and the value of $\gamma$ may be deduced by putting $p=0$.

We must now try to evaluate $f(\mathbb{V})$ and $\chi(\mathrm{E})$.

The transfer of energy due to collisions will be jointly proportional to the number of collisions, and to the mean transfer at each collision. Let us therefore take, in equation 1 ,

$$
\phi(\mathrm{E}, \mathrm{F})=-\iota \sqrt{\mathrm{E}}(\mathrm{F}-3 \mathrm{E}), \quad . \quad .
$$


where $a$ is, for the present, supposed to be independent of $E$ and $\mathrm{F}$.

Suppose that $\mathrm{F}$ is subject to dissipation of amount $\mathrm{eF}$ per unit time, of which a fraction equal to $\theta$ times the whole is regained by absorption of energy from the ather. Suppose, further, that the pulses at collisions are sent out and absorbed in such a way that $\mathrm{F}$ increases, from this cause, at a rate

$$
\sqrt{\mathrm{E}}(\xi \mathrm{F}+\xi \mathrm{E}),
$$

in which the first factor is proportional to the number of collisions, and the second to the mean intensity of pulses.

We may now replace equation (1) by

$$
\frac{d \mathrm{~F}}{d t}=-\alpha \sqrt{\mathrm{E}}\left(\mathrm{F}-{ }_{3}^{1} \mathrm{E}\right)-\epsilon(1-\theta) \mathrm{F}+\sqrt{\mathrm{E}}(\xi \mathrm{F}+\zeta \mathrm{E})
$$

and in the steady state

$$
\begin{array}{r}
\mathrm{F}=f(\mathrm{E})=\frac{\mathrm{E}_{2}^{\frac{3}{2}}\left({ }_{3}^{1} \alpha+\zeta\right)}{(\alpha-\xi)^{N^{\frac{1}{2}}}+\epsilon(1-\theta)} \\
x=\frac{\epsilon(1-\theta)}{(\alpha-\xi) \mathrm{E}^{\frac{1}{2}}+\epsilon(1-\theta)},
\end{array}
$$

we easily find that

$$
\begin{aligned}
& \chi(\mathrm{E})=\frac{\epsilon(1-\theta)}{x}, \\
& f^{\prime}(\mathrm{E})=\frac{1_{3} \alpha+\zeta}{\alpha-\xi}(1-x)\left(1+\frac{1}{2} x\right) .
\end{aligned}
$$

As $\mathrm{E}$ increases from 0 to $\infty, x$ decreases from unity to zero, so that $f^{\prime}(\mathrm{E})$ starts from zero and continually increases to the value $(1 \alpha+\zeta) /(\alpha-\xi)$. The effect of the "lag" in $F$, when small, may be measured by $f^{\prime}(\mathrm{E}) / \chi(\mathrm{E})$, and therefore by

$$
x(1-x)\left(1+\frac{1}{2} x\right),
$$

a quantity which vanishes when $\mathrm{E}=0$.

We therefore see that at low temperatures $\gamma$ will approximate to the value which would be found for it on the assumption that internal vibrations did not exist, namely,

$$
\gamma=1+\frac{2}{3+n},
$$

and as the temperature increases, the value of $\gamma$ will continually increase until it reaches the value which would be 
found for it upon the assumption of $\epsilon=0$. Also as $\mathbf{E}$ increases, the influence of the "lag" in the energy of subsidiary vibrations will increase, being nil when $\mathrm{E}=0$, and infinite when $\mathrm{E}=\infty$.

\section{Summary and Conclusion.}

$\$ 9$. From an inspection of the results which have been obtained, the following general conclusions may be drawn:-

(i.) The value of $\gamma$ is not restricted to having a value given by the formula $1+2 / n$. It will, however, have snch a value if the internal (i.e. vibratory) energy is vanishingly small.

(ii.) When the value of $\gamma$ is different from a value given by the formula $1+2 / n$, the value will, in general, vary with the temperature. In general $\boldsymbol{\gamma}$ will decrease as the temperature increases.

We may conveniently refer to the two kinds of gases mentioned in (i.) and (ii.) as "regular" and "irregular." For a completely regular gas, $\boldsymbol{\Gamma}$ and $\boldsymbol{\gamma}$ become identical, so that the true value of $\gamma$ will be found from sound-observations, and there will be no damping of sound caused by the "lag" in subsidiary degrees of freedom.

(iii.) The generil value for $\gamma$ is

$$
\gamma=1+\frac{2}{3+n+3 \Sigma f^{\prime}(E)}, . . . .
$$

where the summation extends over all subsidiary degrees of freedom.

$\$ 10$. It appears that air is almost completely regular. The value of $\gamma$ is almost exactly $1 \frac{2}{5}$, and this same value is found by either method of experiment *. Leduc's values for: $\gamma$ at temperatures of $0^{\circ}$ and $100^{\circ}$ are $\dagger$

$$
\gamma=1.4040 \quad 100^{\circ} .
$$

The value of $\gamma$ it will be noticed is slightly in excess of the value $1 \frac{2}{5}$, whereas we should certainly expect $\Sigma f^{\prime}(\mathrm{E})$ to be positive. The explanation probably lies in the fact that the rotations of the molecules are not entirely free from reaction with the æther, so that $n$ in equation (24) must be slightly less than 2.

As other instances of regular gases may be mentioned

* Rayleigh, 'Theory of Sound,' $\$ 246$.

$\dagger$ Comptes Rendus, exxvii. p. $66 i$. 
oxygen, hydrogen, nitrogen, and carbon monoxide, all diatomic*.

As an instance of an irregular gas we may take carbon dioxide. Leduc's values $\dagger$ of $\boldsymbol{\gamma}$ are

$$
\gamma=1.3190 \quad 1.2827 .
$$

The value of $\gamma$ for this gas has also been determined by Capstick $\ddagger$. As a result of sis experiments, all at a temperature of about $15^{\circ}$, he finds the value $\gamma=1 \cdot 308$. The variation with temperature is therefore very marked. These results. are all arrived at by measurements on the velocity of sound. As regards the value of $y$ obtained by direct methods we may quote Capstick \$: "The accepted value of $\boldsymbol{y}$ for carbon dioxide obtained by Kundt's method is abont 1·3. Regnault': value for $C_{p}$ between $15^{\circ}$ and $100^{\circ}$ is $\cdot 2025$. Joly's value for $\mathrm{C}_{v}$ between $12^{\circ}$ and $100^{\circ}$ is 1653 , and the ratio of these is $1 \cdot 22$. Both $\mathrm{C}_{p}$ and $\mathrm{C}_{v}$ are difficult to measure accurately, but 7 per cent. seems to be too much to attribute to experimental error. It would seem that we have here to do with forms of vibration that only take their share of the energy, when given more time than is afforded by sound vibrations."

As other instances of irregular gases may be mentioned the gases $\mathrm{N}_{2} \mathrm{O}, \mathrm{NH}_{3}, \mathrm{C}_{2} \mathrm{H}_{4}$, and possibly $\mathrm{C}_{2} \mathrm{H}_{6} \mathrm{O}$ and $\mathrm{O}_{3} \mathrm{H}_{8} \mathrm{O}_{2}$.

In many gases the variation of $\gamma$ with temperature is complicated by chemical dissociation. It is probably for this reason that methyl ether and methylal show an increase of $\gamma$ with temperature.

\$11. It appears that, to reconcile our theory with experiment, it is necessary to suppose that the numerical values of the quantities concerned are such as to make $f^{\prime}(\mathrm{E})$ very small for a subsidiary degree of freedom, at any rate at normal temperatures. Hence, from equation (23), $\epsilon(1-\theta)$ must be large compared with $(\alpha-\xi) \sqrt{\mathrm{E}}$, and the value of $f^{\prime}(\mathbb{E})$ becomes $3 \sqrt{\mathrm{E}}\left(\frac{1}{3} \alpha+\xi\right) / \epsilon(1-\theta)$. If we neglect the "pulses" sent out at collisions, the radiation is of amount $F \in(1-\theta)$ or $\mathrm{E}^{3}\left(\frac{1}{3} a+\zeta\right)$. There is no difficulty in supposing $\zeta$ to be so small as to be negligible, but the case is different as regards $\alpha$.

* Tables giving lists of values of $\gamma$ will be found in Meyer's ' Kinetic Theory of Gases,' p. 125, and also in a paper by J. W. Capstick, 'Science Progress,' iii. p. 10.

† Leduc, l.c. ante.

J. W. Capstick, Phil. Trans, clxxxvi. p. 581.

J. W. Capstick, 'Science Progress,' iii. p. 19. 
Moreover, the radiation at a given temperature would be proportional to $\mathrm{E}^{\frac{3}{2}}$, and this is not in agreement with experiment.

$\$ 12$. Now two molecules must repel one another as soon as their centres are at a sufficiently small distance apart. In nature, this repulsion must vary continuously with the distance apart. Let us, as a first approximation to a representation of this, suppose each molecule surrounded by an imaginary sphere $S$, such that an amount of work $\Omega$ must be expended in bringing the spheres of two molecules into contact. Let us make the assumption that the internal vibrations of two molecules are affected by collision to an appreciable extent, only when the spheres of the two molecules intersect.

At low temperatures, only a very small fraction of the whole number of molecules will possess sufficient energy to penetrate at collision to the surrounding spheres of other molecules, and therefore the mean energy of internal vibration will be affected by collisions only to an infinitesimal extent. It can be shown * that our present assumptions can be represented mathematicaliy by supposing $\alpha$ in equation (22) to contain a factor of the form $e^{-3 \Omega / E}$. Thus it appears that the reconciliation of theory with experiment depends mainly on the possibility of attributing to $\Omega$ a sufficiently great value $t$. If we may regard $\Omega$ as large in comparison with the value of $\mathbf{E}$ at normal temperatures, then the disturbance of $\mathrm{F}$ at collisions will be infinitesimal. Any internal vibrations which may be excited are dissipated by radiation into the ather. As soon as we reach temperatures at which $E$ becomes comparable with $\Omega$, the increase of $F$ caused by collisions becomes appreciable, and as a consequence both $\mathrm{F}$ and the amount of radiation will become appreciable. It appears, therefore, that the point at which $\mathrm{E}$ becomes comparable with $\Omega$ will be the point at which the gas becomes incandescent.

In equation (23), a must now be regarded as a function of $E$. Differentiating this equation logarithmically we shall

* 'Distribution of Molecular Energy,' $\$ 29$.

+ We must, in fact, suppose the vibratory mechanism of a molecule to be sheltered by a tield of repulsive force. Thus the "effective size" of a molecule will be large compared with the dimensions of the vibratory structure. This view fiuds a certain amount of confirmation in the diserepancy between the two values of the radius of an atom found (i.) by kinetic theory methods $\left(10^{-i} \mathrm{~cm}\right.$.), and (ii.) by methods of ionization $\left(10^{-9} \mathrm{~cm}\right.$.). 
M. E. H. Amagat on the Isothermals of Fluids.

find in $\frac{1}{\mathrm{~F}} \frac{d \mathrm{~F}}{d \mathrm{E}}$ additional terms of the form $\frac{1}{\alpha} \frac{d \alpha}{d \mathrm{E}}$ or $\frac{h \Omega}{\mathrm{E}^{2}}$. These terms may be very large when $E$ is small compared with $\Omega$, so that $f^{\prime}(\mathrm{E})$ may be appreciable for values of $\mathrm{E}$ for which $F$ is vanishingly small. In other words, the variation of $\gamma$ with temperature ought, in general, to be perceptible at temperatures below the point of incandescence.

LXI. Note on tive Isothermals of Fluids, and in particular of Hydrogen. By M. Е. H. AMAgAT" *

TN an interesting paper which Mr. Rose-Innes has recently published (Phil. Mag. July 1901) relative to the scale of absolute temperatures this author remarks that the isothermals of hydrogen, which within the limits of temperature and pressure of my experiments appeared sensibly straight and parallel, should in reality only follow this law approximately.

To avoid any misconception I desire to make a few remarks on this subject. The results which Mr. Rose-Innes cites are those of my memoir of 1881 ; the isothermals in this research reached only 400 atmospheres; in these limits and at the temperatures at which I worked the curvature of these lines could not be detected with certainty, but in the much more extended research which I published in 1893 , and in which the superior limit of pressure was raised to 3000 atmospheres, I have returned to this point and have insisted at length on this fact, viz.: the isothermals for all bodies, including hydrogen, show a perfectly distinct curvature, but one which can only be determined with certainty by prolonging these lines much farther than was done in my previous research. The following are the words of the passage referring to this point (Annales de Chimie et de Plysigue):-

"La forme des isothermes au dela de l'ordonnée minima est un des points que je m'étais surtout proposé d'étudier; ces courbes à partir d'une distance d'autunt plus petite de l'ordonnée en question que la température est plus basse, m'avaient paru se transformer en lignes sensiblement droites; j'avais renurqué les indices d'une legère concavité, mais à peine indiquée et $j$ 'avais pensé que le réseau suffisemment prolongé se transformerait pen à peu en un faisceau de droites parallèles. Cette hypothèse était d'autant plus seduisante, que le coefficient angulaire de ce faisceau donnait de suite le

* Communicated by the Author. 